\title{
Modelling of stratified atmospheres of CP-stars
}

\author{
F. LeBlanc and D. Monin \\ Département de physique et d'astronomie, Université de Moncton, Moncton, NB, E1A 3E9, \\ Canada \\ e-mail: leblanfn@umoncton.ca
}

\begin{abstract}
Several observational anomalies seem to confirm the presence of abundance gradients as a function of depth in different types of chemically peculiar stars. Results emanating from the construction of model atmospheres that take into account the abundance gradients caused by radiative diffusion will be presented. The atmospheric structure, which is calculated selfconsistently along with the abundance gradients, will be compared to models with homogeneous abundances. Recent improvements brought to these models will be discussed, along with the intricacies of these calculations and the remaining uncertainties. Several possible applications of such models will also be presented.
\end{abstract}

Keywords. Diffusion, stars: abundances, stars: atmospheres, stars: chemically peculiar

\section{Introduction}

Mounting observational evidence seem to show that element stratification is present in the atmospheres of several types of stars. For atomic diffusion (Michaud 1970) to be able to create abundance stratifications, an hydrodynamically stable atmosphere is required. The accumulation (or depreciation) of the elements as a function of depth will modify the atmospheric structure of such stars. It is then imperative, not only to obtain the stratification profiles of the various elements, but also to include the back effect of these abundance gradients on the structural calculation of the atmosphere. Up until now, the vast majority of photometric and spectroscopic studies have employed homogeneous models.

Recently, model atmospheres including stratification due to diffusion have been constructed for white dwarf stars (Dreizler \& Wolff 1999) and horizontal-branch stars (HuiBon-Hoa et al. 2000). These models were successful in explaining certain observational anomalies. For example, for white dwarf stars, it was shown that the models with diffusion could explain a flux depression in the ultraviolet (see Fig. 6 of Dreizler \& Wolff 1999). Meanwhile, the observed photometric jumps (e.g., Grundhal et al. 1999) and gaps (e.g., Caloi 1999) that are observed in hot horizontal-branch stars are qualitatively reproduced by stratified models (see Fig. 1 and 2 of Hui-Bon-Hoa et al. 2000), assuming that diffusion becomes dominant in stars hotter than $T_{\text {eff }} \simeq 11,500 \mathrm{~K}$. For more details concerning the observed anomalies of horizontal branch stars see Moehler (2005).

Since stratification seems to be present in Ap stars (e.g., Wade et al. 2001, Babel 1994) and might also be present in HgMn stars (e.g., Savanov \& Hubrig 2003), selfconsistent model atmospheres including diffusion are urgently needed to properly study these stars (for a review of observational evidence of stratification in stellar atmospheres see Ryabchikova et al. 2003). In this paper we will present results of recent modelling of the atmospheres of an A type star with such self-consistent models. These models are calculated with an improved version of the Hui-Bon-Hoa et al. (2000) models, which 
are based on the PHOENIX (e.g., Hauschildt et al. 1999) code. We will briefly review the method and ingredients used in our models as well as describing the improvements recently included. Examples of such models will be shown and future applications, actual limitations and possible improvements will be discussed.

\section{Theory}

The diffusion velocity $\left(V_{i}\right)$ of an ion $i$ of a trace element $A$ can be approximated by (Burgers 1960, Vauclair \& Vauclair 1982, Alecian \& Vauclair 1983, Landstreet et al. 1998):

$$
V_{i} \approx D_{i}\left[-\nabla \ln C_{i}+\frac{A_{i} m \mathrm{p} g_{\mathrm{rad}}}{k T}-\left\{\left(A_{i}-1\right)+\left(A_{i}-Z_{i}\right) f_{\mathrm{p}}\right\} \nabla \ln P+\kappa_{T} \nabla \ln T\right]
$$

Here $D_{i}$ is the diffusion coefficient, $C_{i}$ is the concentration of the ion, $A_{i}$ its atomic mass, $g_{\mathrm{rad}}$ its radiative acceleration, and $Z_{i}$ its ionic charger. The local temperature, pressure and ionization fraction of $\mathrm{H}$ II are designated by $T, P$ and $f_{\mathrm{p}}$. The factor $f_{\mathrm{p}}$ appears to give the proper asymptotic values of the electric field in the medium (e.g., Landstreet et al. 1998). The term including $\nabla \ln T$ is the thermal diffusion term, which was neglected in our calculations since it is negligible in the atmosphere. The abundance gradient term was also neglected in the models presented here.

The most important ingredient in the diffusion equation is $g_{\mathrm{rad}}$. Contrarily to diffusion calculations in stellar interiors (e.g., Turcotte et al. 1998) where the radiative flux used is the one given by the so-called diffusion approximation (Milne 1927) which is only valid at large optical depths, in the atmosphere the flux must be explicitly calculated by resolving the radiative transfer equation at a large number of frequency points. The $g_{\text {rad }}$ values used here are thus calculated using the opacity sampling method (e.g., LeBlanc et al. 2000) which is the preferred calculation method to properly evaluate line blending and saturation effects.

A major source of uncertainty in $g_{\mathrm{rad}}$ is related to the possible redistribution of momentum among the ions (e.g., Gonzalez et al. 1995). Since the various ions have different mobility (or diffusion coefficients), if an ion that acquires momentum following a boundbound transition, for example, ionizes or recombines before losing this momentum, the acceleration can thus be modified. Since the appropriate evaluation of this redistribution effect is extremely difficult, this causes an uncertainty in $g_{\text {rad }}$. This is particularly important in the upper atmosphere since there is a large difference in the diffusion coefficients of the neutral state as compared to the once ionized species. In the calculations presented here, we will approximate the redistribution effect with the method used in Hui-Bon-Hoa et al. (1996) which is based on the formalism described by Montmerle \& Michaud (1976).

Magnetic fields can also affect atomic diffusion. First, the diffusion of charged ions is modified when they cross magnetic field lines. Also, the Zeeman effect can also change the value of the radiative accelerations (Alecian \& Stift 2004). Neither of these magnetic effects are included in the models presented here.

\section{Modelling and results}

Our model atmospheres try to simultaneously calculate the abundance stratifications of various elements and the atmospheric structure. We thus modify the abundance of each element at each depth in the atmosphere to converge the diffusion velocity to zero (i.e., equilibrium abundances). The magnitude of the change brought to the abundance is a 
function of the diffusion velocity, and is greater where the velocity is higher. The diffusion velocities obtained in a previous iteration are used to calculate correction factors. The abundances are then changed by these factors.

An iteration scheme that alternates between abundance and temperature corrections is used. The atmospheric physical structure has to be adjusted each time the abundances are changed. It is necessary to iteratively converge the diffusion velocity towards zero first, and then the physical structure to insure that these structures are self-consistent. A series of six abundance corrections is followed by a series of temperature corrections. We repeat this several times or until we see successful convergence. The robustness of the scheme depends on the number of abundance iterations in a series. Changes in the physical structure must be slow enough to ensure proper convergence. About 10 to 15 temperature iterations are typically required to reach convergence to better than a few $\mathrm{K}$. We find that this iteration approach provides excellent convergence speed and stability.

The radiative transfer equation is solved before each abundance correction or temperature correction iteration. The computing time to obtain a converged model is considerable since to obtain precise $g_{\text {rad }}$ the radiative transfer equation is solved at almost a quarter of a million frequency points.

The elements included in the PHOENIX code are $\mathrm{H}-\mathrm{Ga}, \mathrm{Kr}-\mathrm{Nb}, \mathrm{Ba}$ and La. The line atomic data used are from Kurucz (1994). The models presented here are plane-paralel atmospheres that are calculated in LTE. Both bound-bound and bound-free transitions are included in the $g_{\mathrm{rad}}$ calculations.

Several improvements to the models presented in Hui-Bon-Hoa et al. (2000) have been added to the atmospheric code. Most are related to that they supposed a completely ionized hydrogen medium. Since we now use this code for cooler stars, such as A-type stars, it was necessary to more precisely evaluate diffusion in a partially ionized buffer gas. Changes were thus made to the diffusion coefficients and to the equation of the electric field that appears in the diffusion equation. These forced us to use a different convergence scheme to modify the abundances to obtain null diffusion velocities. The code was also modified to evaluate the effect of mass loss on the abundance stratification of the elements.

\subsection{Stratification profiles}

The abundance of a given element that can be supported by radiative pressure varies as a function of depth since different ions have different opacities and thus different $g_{\mathrm{rad}}$ values. Stratification profiles can then be very different from one element to another.

Wade et al. (2001) found that they could better fit the observed lines of several elements with a two zone empirical stratification model for the Ap star $\beta \mathrm{CrB}$. They found that for $\mathrm{Ca}, \mathrm{Fe}$ and $\mathrm{Cr}$, that their lines are better fitted with a strong underabundance in the outer atmosphere and an overabundance in deeper layers, than with a vertically homogeneous abundance. It should be noted that the underlying atmospheric model used was a homogeneous model and the two zone stratification profile was only used for their line synthesis. They found that the transition zone for the elements considered (Ca, Fe and $\mathrm{Cr}$ ) for the Ap star $\beta \mathrm{CrB}$ is found at $\log \left(\tau_{5000}\right) \simeq-0.7$.

Figure 1 shows the one step abundance profiles for $\mathrm{Fe}$ and $\mathrm{Cr}$ found by Wade et al. (2001) as compared to the ones found in various self-consistent models for $\beta \mathrm{CrB}$. We chose $T_{\text {eff }}=7700 \mathrm{~K}$ for $\beta \mathrm{CrB}$ while realizing that uncertainties exist in determining $T_{\text {eff }}$ for Ap stars. The abundance increases in our model assuming no convective mixing are deeper than those found by Wade et al. (2001). In the models shown, the abundance gradient term in the diffusion equation was neglected. However, preliminary results show that this term cannot account for the deeper increase of the abundances in the model 


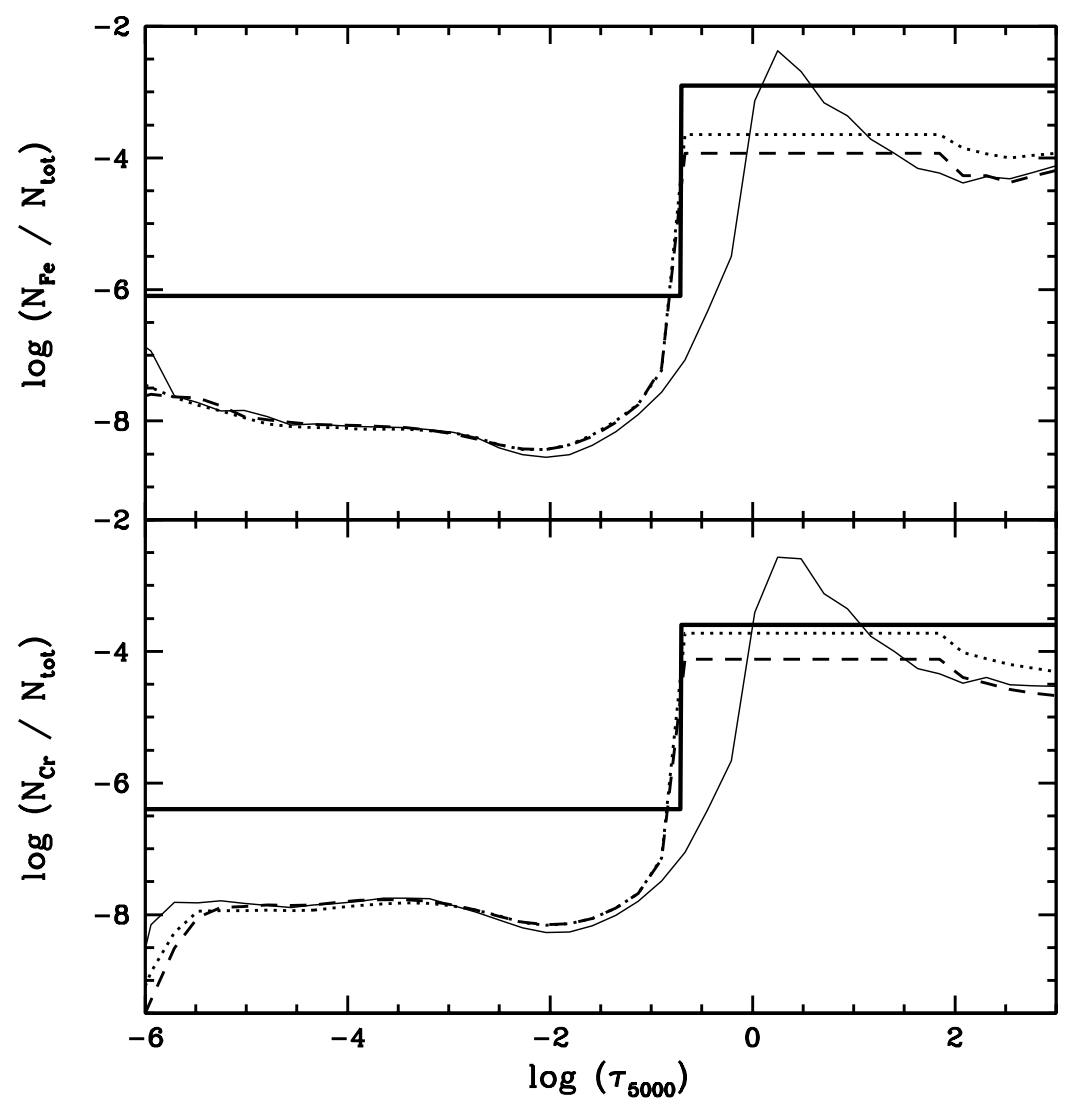

Figure 1. Abundance profiles of $\mathrm{Fe}$ and $\mathrm{Cr}$ in a $T_{\text {eff }}=7700 \mathrm{~K}$ model as a function of optical depth at $5000 \AA$. The heavy solid line is the empirical profile of Wade et al. (2001) found for $\beta \mathrm{CrB}$, the thin solid line is the profile in a self-consistent model with no convective mixing, the long dashed line is the profile in a self-consistent model with convective mixing, while the dotted line is the profile in a model with convective mixing and a mass loss rate of $3 \times 10^{-15} M_{\odot} \mathrm{yr}^{-1}$.

with no convective mixing from those found in observations. It should be noted that in the theoretical models, this depth depends on the $T_{\text {eff }}$ of the model.

Since Wade et al. (2001) found that the abundance jumps abruptly at $\log \left(\tau_{5000}\right) \simeq-0.7$ for the three elements studied and this is where one would expect for convection to begin, according to the Schwarzschild criterion, therefore we calculated models in which convective mixing was present. In these models we supposed that the presence of a strong magnetic field suppresses overshooting since this would erase elemental stratification. Indeed, $\beta \mathrm{CrB}$ possesses a strong magnetic field of $5 \mathrm{kG}$ (Mathys et al. 1997). Even though it is widely believed that the presence of magnetic fields at least partially suppresses convection, it could be possible for weak convective mixing to persist and dominate atomic diffusion. A convection velocity of the order of $1 \mathrm{~cm} \mathrm{~s}^{-1}$, which is well below the detection treshold, is sufficient to dominate diffusion where a convection zone could possibly persist. The supported abundance in the convection zone was taken as the abundance sustained by radiative pressure at its bottom. The abundance profiles are then similar to the two plateau model used by Wade et al. (2001). Figure 1 shows the stratification in models with a convective mixing zone. It should be noted that the abundance supported in the convective zone is very sensitive to the position of the bottom of this zone since 

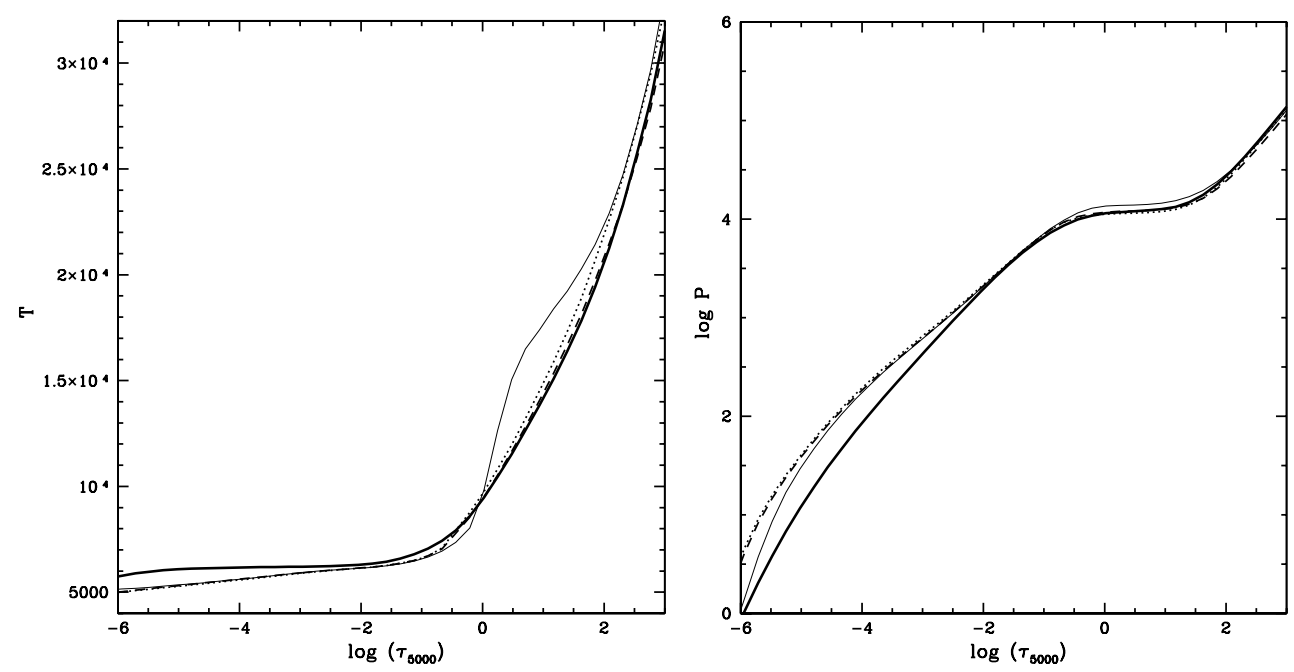

Figure 2. Temperature and pressure in a $T_{\text {eff }}=7700 \mathrm{~K}$ model as a function of optical depth at $5000 \AA$. The heavy solid line is for an homogeneous model, the thin solid line is for a self-consistent model with no convective mixing, the long dashed line is for a self-consistent model with convective mixing, while the dotted line is for a self-consistent model with convective mixing and a mass loss rate of $3 \times 10^{-15} M_{\odot} \mathrm{yr}^{-1}$.

radiative accelerations can vary quickly as a function of depth there. These results seem to show that in Ap stars, some slow convective mixing might persist. As for the abundance supported at shallow layers, our results are quite different from those found by Wade $e t$ al. (2001).

Babel (1992) calculated the stratification of Ca in an Ap star, but without calculating the atmospheric structure. His model included a convective mixing zone. He showed that the value of the upper plateau strongly depends on mass loss. We therefore calculated a model with a mass loss rate of $3 \times 10^{-15} M_{\odot} \mathrm{yr}^{-1}$. We can see in Figure 1 that the mass loss increases the abundance that can be supported in the convective mixing zone for the two elements considered and gives values closer to the empirical stratifications found by Wade et al. (2001).

\subsection{Atmospheric structure of stratified atmospheres}

Figure 2 shows the temperature and pressure as a function of depth for self-consistent models including diffusion with and without convective mixing as compared to a homogeneous stellar atmosphere of a $T_{\text {eff }}=7700 \mathrm{~K}$ star. The abundances used for the homogeneous models are the average abundances observed in $\beta \mathrm{CrB}$ (Ryabchikova, private communication).

We can see that in the diffusion models the temperature is lower in the outer atmosphere, as compared to the homogeneous model, while it is larger deeper in the atmosphere, where large accumulations of the elements occur. This temperature increase is related to the increase in opacity due to accumulation of several elements in these deep layers. The maximum temperature increase occurs at depths larger than $\tau_{5000}=1$. This maximum increase is of the order of $30 \%$ in the model without convective mixing, while it is only approximately $6 \%$ in the model with convective mixing and mass loss.

Meanwhile the pressure in the outer atmosphere is up to two to three times larger in the models with diffusion as compared to the homogeneous model. This is due to that 
many elements are very underabundant there making the real physical depth larger for the same optical depth in the models with stratification. In deep layers, the pressure difference between the homogeneous model and the stratified model with convective mixing and mass loss is less than $7 \%$.

\section{Conclusion}

The results from modelling which include the stratification of the elements due to diffusion have shown that the stratification profiles found are similar to those observed in the Ap star $\beta \mathrm{CrB}$, but convection mixing is needed to get better fitting. It should be noted, that in the models with convective mixing, the value of the upper abundance plateau is very sensitive to the depth of the convection zone and also depends on the mass loss rate. Stratification affects the physical structure of the atmosphere moderately in the models with convective mixing, and stongly in the models assuming no convective mixing. However, the modelling of magnetic stars is quite difficult since abundances patches are observed on their surface. Therefore, one dimensional models like those presented here can only have limited success in reproducing observational anomalies related to the accumulation (or depreciation) of the various elements in the atmospheres of magnetic stars.

The effect of the magnetic field on diffusing atoms as well as that of the Lorentz force on the structure of the atmosphere and the interaction of the diffusing atoms with the magnetic field could have an important effect on the atmosphere (Valyavin et al. 2004, LeBlanc et al. 1994). Missing opacity sources, like those of rare-earth elements which are very overabundant in Ap stars, should also be included in future modeling. The interaction between the atmosphere and the interior, during the diffusion process, as well as the possible importance of the initial conditions could also play an important role in the final state of the atmosphere. Other physical phenomena such as light-induced drift (Atutov \& Shalagin 1988, LeBlanc \& Michaud 1993) should also be included in HgMn models to attempt to explain certain observed isotopic anomalies (Dworetsky \& Vaughan 1973).

The results shown here in which the elemental stratification is calculated self-consistently with the atmospheric structure in CP-stars are encouraging. However, many other physical phenomena that were neglected here and mentioned above can come into play. Much more work needs to be done in modelling stratified atmospheres to elucidate some of the many observational anomalies observed in CP-stars. Also, abundance stratifications derived for a greater number of Ap stars such as those presented by Ryabchikova et al. (2005) will also enable a better constraint of the models.

\section{References}

Alecian, G., \& Vauclair, S. 1983, Fund. of Cosmic Physics, 8, 369

Alecian, G., \& Stift, M. J. 2004, A\&A, 416, 703

Babel, J. 1992, A\&A, 258, 449

Babel, J. 1994, A\&A, 283, 189

Burgers, J.M. 1960, in Plasma Dynamics, ed. F.H. Clauser (Reading: Addison-Wesley), 119

Caloi, V. 1999, A\&A, 343, 904

Dreizler, S., \& Wolff, B., 1999, A\&A, 348, 189

Dworetsky, M. M., \& Vaughan, A. H., 1973, ApJ, 181, 811

Gonzalez, J.-F., LeBlanc, F., Artru, M.-C., \& Michaud, G. 1995, A\&A, 297, 223

Grundahl, F., Catelan, M., Landsman, W. B., Stetson, P. B., \& Andersen, M. I. 1999, ApJ, 524, 242 
Hauschildt, P. H., Allard, F., \& Baron, E. 1999, ApJ, 512, 377

Hui-Bon-Hoa, A., Alecian, G., \& Artru, M.-C., 1996, A\&A, 313, 624

Hui-Bon-Hoa, A., LeBlanc, F., \& Hauschildt, P.H., 2000, ApJ, 535, L43

Kurucz, R. L., 1994, Atomic Data for Opacity Calculations (Kurucz CD-ROM No.1)

Landstreet, J. D., Dolez, N., \& Vauclair, S. 1998, A\&A, 333, 977

LeBlanc, F., \& Michaud, G., 1993, ApJ, 408, 251

LeBlanc, F., Michaud, G., \& Babel, J. 1994, ApJ, 431, 388

LeBlanc, F., Michaud, G., \& Richer, J. 2000, ApJ, 538, 876

Mathys, G., Hubrig, S., Landstreet, J.D., Lanz, T., \& Manfroid, J., 1997, A\&ASS, 123, 353

Montmerle, T., \& Michaud, G., 1976, ApJS, 31, 489

Michaud, G., 1970, ApJ, 160, 641

Milne, E.A., 1927, M. N., 87, 687

Moehler, S., 2005, These Proceedings, 395

Ryabchikova, T.A., Leone, F., Kochukhov, O., \& Bagnulo, S., 2005, These Proceedings, DP1

Ryabchikova, T.A., Wade, G.A., \& LeBlanc, F., 2003, in Modelling of Stellar Atmospheres, ed.

N. Piskunov, W.W. Weiss \& D. F. Gray, IAU Symp. 210, 301

Savanov, I., \& Hubrig, S., 2003, A\&A, 410, 299

Turcotte, S., Richer, J., \& Michaud, G., 1998, ApJ, 504, 559

Valyavin, G., Kochukhov, O., \& Piskunov, N., 2004, A\&A, 420, 993

Vauclair, S., \& Vauclair, G. 1982, ARA\&A, 20, 37

Wade, G. A., Ryabchikova, T. A., Bagnulo, S., \& Piskunov, N. 2001, in Magnetic Fields across the Hertzsprung-Russell Diagram, ed. G. Mathys, S. K. Solanki \& D. T. Wickramasinghe, ASP Conf. Series 248, 373

\section{Discussion}

TALON: How unique is the solution you calculate?

LEBLAnC: Diffusion phenomena is dependant on the initial conditions since for example, blending effects, which are included in our calculations, modify the radiative accelerations of the elements. We suppose that initially, the atmosphere is homogeneous. Tests show that the final solution depends weakly on the initial atmosphere used provided that the starting model is 'reasonable'.

VAUCLAIR: Large accumulation of heavy elements in the outer layers of stars create an inverse $\mu$-gradient which is unstable and leads to a double-diffusive or thermohaline convectio). The models should include such physical process in the future.

LEBLANC: I agree that the large overabundances obtained, for example in the models assuming no convective mixing, could lead to unstabilities and will have to be investigated.

MATHys: It is an excellent thing that, on the theoretical side, you treat element stratification with self-consistent models. We should be careful that on the observational side, much less consistency is achieved. We know (e.g., from the core-wing anomaly in Balmer lines of hydrogen) that standard model atmospheres do not adequately represent the atmosphere of Ap stars). Yet we use such a model to find different abundances from different lines of a given ion and conclude from there to the existence of vertical stratification of the ion. Without necessarily questioning the latter, this calls for caution against overinterpreting the quantitative details of the abundance gradient that are currently derived. 
LEBLAnC: The observed abundance stratification shown here as compared to those obtained in the self-consistent model atmospheres are only indicative. As mentioned in several talks, the abundances and even the value of the effective temperatures of Ap stars are very difficult to evaluate precisely.

WADE: The situation is indeed quite bad (notice there were no error bars on the empirical stratification distribution). Not only is the temperature a 'free parameter', but we can study stratification best in those stars for which the least information about other complications (e.g., surface abundance + magnetic field structures). Investigations are underway to try to understand the influence of these factors. 